\title{
A Word From the Editor
}

This is the 30th anniversary of the TESL Canada Journal, which was established in 1984 and has published a wide range of articles of relevance to the teaching of English as a second language since then. Now is, therefore, an appropriate time to express appreciation to all the journal staff members (editors, reviewers, and others without whom the journal would not be possible), as well as to all those who have made the journal such a success over the years.

In this Spring 2014 issue, we continue to offer a range of articles of relevance to teacher educators, ESL instructors and learners, academics, researchers, and graduate students within and beyond Canada. Caroline Payant's research article on the incorporation of video-mediated reflective tasks in MATESOL programs will be of interest to teacher educators and the preservice and in-service teachers in their programs, native- and non-native speakers alike. Kent Lee and Leila Ranta's article on the use of Facebook by international students offers insights into the relationship between social media and Canadian and other post-secondary students; it also provides recommendations for teachers interested in integrating social media into their classroom instruction. Lijuan Ye's comparative genre study of spoken argumentation by Chinese and native speakers of English offers pedagogical implications and suggestions for future research in this area.

An increasing number of ESL students use cellphones, both within and outside the classroom. Jeff Brown's Perspectives article provides a framework for reflection by instructors whose students use cellphones; it should be of interest to teachers in the ESL classrooms of both today and the future. Iryna Lenchuk and Amer Ahmed have responded to Brett Reynold's article, "Determiners, Feline Marsupials, and the Category-Function Distinction: A Critique of ELT Grammars," which was published in the Spring 2013 issue of the TESL Canada Journal. Brett Reynolds has kindly provided a reply to their article, and both articles are likely to generate thoughtful consideration of the complexities of grammar texts and English grammar pedagogy.

In the Book Review section of this issue, Marilyn Lewis has reviewed Social Networking for Language Education, a topic of ongoing interest in the field. Jennifer MacDonald's review of Academic Inquiry: Writing for Post-secondary Success will similarly be of interest to ESL instructors and students in English language classrooms.

Thank you to all who have contributed in various ways to this issue of the TESL Canada Journal. Our next issue will be the 2014 Special Issue on TaskBased Language Teaching and Learning, guest edited by Dr. Bill Dunn. We look forward to continuing to receive stimulating manuscripts and reviews from you and your colleagues as we head into our 31st year of publication!

Marian Rossiter 


\section{Un mot de l'éditrice}

Cette année, la Revue TESL du Canada célèbre ses 30 ans. Fondée en 1984, la revue publie une vaste gamme d'articles portant sur l'enseignement de l'anglais comme langue seconde. Le moment est donc approprié pour exprimer notre reconnaissance à tous les membres du personnel de la revue (éditeurs, réviseurs et toutes les autres personnes sans lesquelles le journal ne pourrait exister), ainsi qu'à tous ceux qui ont contribué au succès de la revue au fil des années.

Dans ce numéro du printemps 2014, nous continuons à offrir un éventail d'articles susceptibles d'intéresser les formateurs d'enseignants, les enseignants et les apprenants d'ALS, ainsi que les chercheurs, les professeurs et les étudiants aux cycles supérieurs au Canada et ailleurs. L'article de Caroline Payant porte sur l'intégration, dans les programmes de MATESOL, de tâches réflexives effectuées par vidéo; sa recherche saura intéresser les formateurs, les enseignants en formation et en poste, qu'ils soient de langue maternelle ou allophones. Kent Lee et Leila Ranta offrent, dans leur article traitant de l'emploi de Facebook par des étudiants internationaux, un regard sur le rapport entre les médias sociaux et les étudiants à l'université, tant canadiens qu'étrangers. De plus, ils présentent des recommandations aux enseignants qui voudraient intégrer les médias sociaux dans leur pédagogie. Une étude comparative par Lijuan Ye décrit l'argumentation à l'oral d'étudiants chinois et la distingue de celle d'étudiants de langue maternelle anglaise. L'article conclut par une explication des incidences pédagogiques des résultats ainsi que des suggestions de pistes pour la recherche dans le domaine à l'avenir.

L'emploi des téléphones cellulaires s'accroit auprès des étudiants en ALS, dans la salle de classe comme à l'extérieur. Dans la section Perspectives, l'article par Jeff Brown présente un cadre de réflexion aux enseignants dont les étudiants se servent de téléphones cellulaires; les observations qui $s^{\prime} y$ trouvent resteront pertinentes pour les enseignants d'ALS pendant des années à venir. Iryna Lenchuk et Amer Ahmed ont réagi à l'article par Brett Reynold intitulé "Determiners, Feline Marsupials, and the Category-Function Distinction: A Critique of ELT Grammars," qui a paru dans le numéro du printemps 2013 de cette revue. Brett Reynolds ayant eu l'obligeance de leur répondre, les deux articles devraient provoquer un examen en profondeur de la complexité de la grammaire anglaise et de son enseignement.

Marilyn Lewis présente un compte rendu portant sur un sujet des plus pertinents et actuels : Social Networking for Language Education. Dans la même section, les lecteurs trouveront une critique par Jennifer MacDonald de Academic Inquiry: Writing for Post-secondary Success, qui saura intéresser tant les enseignants que les étudiants d'ALS. 
Un grand merci à tous et à toutes qui ont contribué à ce numéro de la Revue TESL du Canada. Notre prochain numéro sera le numéro spécial de 2014 et portera sur l'enseignement et l'apprentissage des langues centrés sur les tâches. Dr. Bill Dunn en sera l'éditeur invité. Forts de nos 30 ans de publication, nous attendons avec intérêt les manuscrits et les critiques que nous continuerons à recevoir de vous et de vos collègues!

Marian Rossiter 